\title{
Association between Laterality and Location of Deep Vein Thrombosis of Lower Extremity and Pulmonary Embolism
}

\author{
Sangmin Gong ${ }^{1}$, Eun Ji Lee ${ }^{1}$, Jin Sung Kim', Hyangkyoung Kim², Minsu Noh ${ }^{1}$, Hojong Park ${ }^{1}$, \\ Bong Won Park', Songsoo Yang ${ }^{1}$, and Sang Jun Park ${ }^{1}$ \\ ${ }^{1}$ Department of Surgery, Ulsan University Hospital, University of Ulsan College of Medicine, Ulsan, ${ }^{2}$ Division of Vascular Surgery, \\ Department of Surgery, Asan Medical Center, University of Ulsan College of Medicine, Seoul, Korea
}

Purpose: The aim of this study was to investigate the relationship between the anatomical location of thrombi in the lower extremities and the development of pulmonary embolism (PE).

Materials and Methods: We collected and analyzed the data of patients diagnosed with deep vein thrombosis (DVT) of the lower extremities between 2006 and 2015, and included those whose computed tomography (CT) data were available for PE identification. We evaluated the relationship between the laterality and the proxi$\mathrm{mal} /$ distal location of the thrombi in lower extremites and the location of PE.

Results: CT images were available for 388/452 patients with DVT. After excluding 32 cases with bilateral involvement, 356 cases were included for analysis in this study. The ratio of DVT in the left:right leg was 232:124. PEs developed in $121(52.2 \%)$ patients with left-sided DVT and in 78 (62.9\%) with right-sided DVT $(\mathrm{P}=0.052)$. PEs in the main pulmonary arteries developed in $36(15.5 \%)$ patients with left leg DVT and in $30(24.2 \%)$ with right leg DVT $(P=0.045)$. The most frequent site of thrombosis associated with the development of PE was the left iliac vein (59/199, 29.6\%). According to the anatomical segment of the leg affected by DVT, patients with DVT in the right femoral vein $(50 / 71,70.4 \% ; P=0.016)$ had the highest rate of occurrence of $\mathrm{PE}$.

Conclusion: PE develops more frequently in patients with right-sided DVT than in those with left-sided DVT. Therefore, careful observation for the possible development of PE is recommended in cases with right-sided DVT of the lower extremity.

Key Words: Venous thrombosis, Pulmonary embolism, Mortality, Computed tomography angiography
Received November 19, 2020

Revised March 29, 2021

Accepted April 25, 2021

Published on May 25, 2021

Corresponding author: Sang Jun Park

Department of Surgery, Ulsan University Hospital, University of Ulsan College of

Medicine, 877 Bangeojinsunhwando-ro, Dong-gu, Ulsan 44033, Korea.

Tel: 82-52-250-7109

Fax: 82-52-250-7350

E-mail: sjpark@uuh.ulsan.kr

https://orcid.org/0000-0001-8112-6331

Copyright (c) 2021 The Korean Society for Vascular Surgery

This is an Open Access article distributed under the terms of the Creative Commons Attribution Non-Commercial License (http://creativecommons.org/licenses/by-nc/4.0) which permits unrestricted non-commercial use, distribution, and reproduction in any medium, provided the original work is properly cited.

Vasc Specialist Int $\bullet$ https://doi.org/10.5758/vsi.200075

\section{INTRODUCTION}

Deep vein thrombosis (DVT) can cause significant morbidity and mortality associated with pulmonary embolism (PE) or post-thrombotic syndrome (PTS). Earlier ago, the mainstay of treatment for acute DVT was anticoagulation therapy with heparin and warfarin [1], and the laterality or extent of DVT was not a consideration in the selection of the treatment modality $[1,2]$. Although standard anticoagulation therapy is the mainstay of treatment, the use of catheter-directed thrombolytic therapy (CDT) and percutaneous mechanical thrombectomy can decrease the early problem 
of leg edema and pain and might reduce the development of long-term post-thrombotic sequelae of iliofemoral (IF) DVT $[2,3]$.

May-Thurner syndrome is three-to-eight times more frequent on the left side than on the right [4,5] and accounts for approximately 50\%-60\% of the cases of left $\mathrm{IF}$ DVT [6,7]. This condition is considered as one of the main contributors to the left-sided predominance of DVT in the lower extremity [8-10]; thus, correction of the mechanical compression is often necessary for patients with left-sided DVT to reduce the risk of recurrent DVT and PTS [11]. CDT is indicated only for extensive proximal DVT because of the increased risk of major bleeding events [12]. Therefore, the laterality or proximal extent of DVT needs to be considered before establishing a treatment plan.

Massive PEs associated with DVT can lead to mortality [13]. Acute PE that is not detected and treated promptly is associated with a high rate of mortality [14]. Several assessment tools, such as the Wells score and revised Geneva score, can be used to confirm suspected DVT and PE [15,16]. However, only a few studies have addressed the association between the laterality or the location of DVT and the probability of occurrence of PE. We postulated that the risk of occurrence of PE is relatively higher in patients with rightsided DVT because left-sided DVT is more frequently associated with iliac vein compression, which might prevent embolization of the thrombus. Therefore, the present study aimed to determine the anatomical distribution of DVT and the probability of occurrence of PE based on the distribution of DVT.

\section{MATERIALS AND METHODS}

\section{1) Patients}

We collected the data of patients diagnosed with DVT of the lower extremities from 2006 to 2015 at a single tertiary hospital, and subjects whose computed tomography (CT) images were available for the detection of PE were included in the study. The cases were extracted from our own registry data. The diagnosis of DVT was made by checking the venous compressibility and luminal color filling on Doppler ultrasonography and measuring the serum D-dimer level; levels less than $0.5 \mu \mathrm{g} / \mathrm{dL}$ were considered as indicators of chronic thrombi and were excluded from the study. For cases diagnosed with DVT, CT was performed to check for PE if there was no contraindication, such as allergy to the contrast medium or renal dysfunction. Following the diagnosis, blood samples were obtained to test for thrombophilia, and anticoagulation therapy was administered immediately. In line with the treatment policy of our hospital, thrombolysis, stent placement, and inferior vena cava filter were applied in patients in whom this treatment was indicated. The indications for thrombolysis with or without iliac vein stent were proximal vein thrombosis above the inguinal ligament, age less than 60 years, and duration since the onset of symptoms less than 2 weeks.

Bilateral DVTs were excluded from the analysis of laterality. The location of DVT was classified as right/left and iliac/femoral/popliteal/calf according to the most proximal location of the thrombi. PE was classified according to the involved arteries: main, lobar, segmental, or subsegmental artery (Fig. 1). The correlation between the laterality and the location of DVT and PE was investigated. Compression of the left iliac vein was defined as the left iliac vein being compressed between the right iliac artery and the 5th vertebral body, confirmed by CT.

\section{2) CT imaging}

A uniform protocol was followed for CT pulmonary arteriography and indirect venography using a 256 multidetector scanner (Brilliance iCT; Philips Healthcare, Cleveland, $\mathrm{OH}, \mathrm{USA})$. The $\mathrm{CT}$ pulmonary arteriography technique involved the administration of $100 \mathrm{~mL}$ of iodinated contrast (lomeron; Bracco, Milano, Italy) followed by $50 \mathrm{~mL}$ of normal saline at the rate of $3 \mathrm{~mL} / \mathrm{sec}$ through a cannula in the arm using an automated injector. The patients were placed in the supine position, and a helical CT scan of the thorax was performed during maximal inspiration in a caudocranial direction (100 mA, $120 \mathrm{kV}$ with automated Z-axis dose modulation) with $1.5-\mathrm{mm}$ axial image reconstruction. For image acquisition, scanning of the thorax was scheduled and performed immediately after the injection of the contrast bolus. Indirect CT venography imaging was performed $180 \mathrm{sec}$ after the completion of intravenous contrast injection, and images pertaining to the region extending from the iliac crests to the tibial plateaus were obtained. Noncontiguous axial scanning was performed in a craniocaudal direction (150 mA, $120 \mathrm{kV}$ ) with a 5-mm collimated slice. The CT was evaluated by two radiologists, and the values used in this study were reviewed by two vascular surgeons and recorded based on their consensus.

PE score was calculated to quantify the severity of PE; 4, 3,2 , and 1 points were assigned for $\mathrm{PE}$ in the main pulmonary trunk or artery, lobar arteries, segmental arteries, and subsegmental arteries, respectively (Fig. 1).

\section{3) Data analysis}

Continuous variables (such as PE scores) were compared using the Student t-test or Mann-Whitney U-test, while 

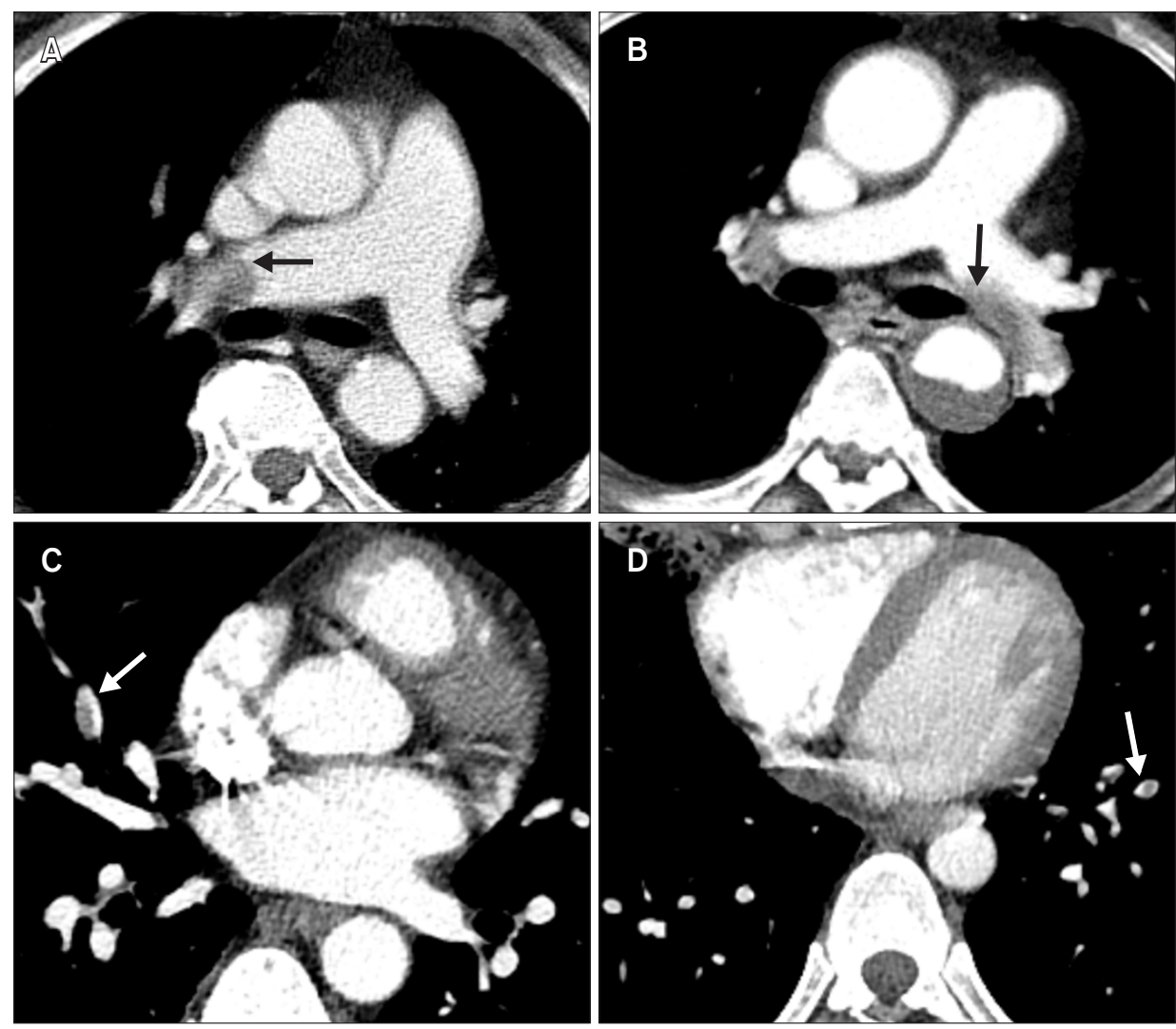

Fig. 1. Classification of pulmonary embolism (PE). PE was classified based on the location of the emboli observed on the computed tomography scan. (A) Main, (B) lobar, (C) segmental and (D) subsegmental PE. Black and white arrows indicate PEs.

categorical variables, including the correlation between DVT and PE, were assessed using the $\chi^{2}$ test or Fisher exact test as appropriate. Descriptive statistical values are presented as means with standard deviations. SPSS ver. 21.0 (IBM corp., Armonk, NY, USA) was used for all statistical calculations. All $\mathrm{P}<0.05$ were considered statistically significant.

\section{4) Ethical considerations}

This study was approved by the Institutional Review Board of Ulsan University Hospital (IRB number: UUH2020-03-053) and complied with the principles of the Declaration of Helsinki. The requirement for informed consent was waived due to the observational nature of the study.

\section{RESULTS}

During the study period, we identified 452 patients with DVT, the CT scans of 388 of whom were available. After excluding 32 cases with bilateral involvement, 356 cases were included in the final analysis. The mean age of the patients was $63.5 \pm 15.6$ years (range, 12-98 years), and 191 patients (53.7\%) were female. There were 232 cases with left-sided DVT (65.2\%) and 124 cases with right-sided DVT (34.8\%). The clinical characteristics of patients with DVT are summarized in Table 1. The duration of anticoagulation therapy
Table 1. Clinical characteristics of patients with deep vein thrombosis $(n=356)$

\begin{tabular}{lc}
\hline \multicolumn{1}{c}{ Clinical characteristic } & Number \\
\hline Age (y) & $64(12-98)$ \\
Sex, female & $191(53.7)$ \\
Immobilization & $96(27.0)$ \\
Surgery related & $78(21.9)$ \\
Malignancy related & $69(19.4)$ \\
Trauma related & $27(7.6)$ \\
Thrombophilia & $93(26.1)$ \\
Oral contraceptive & $5(1.4)$ \\
Inferior vena cava filter insertion & $77(21.6)$ \\
Invasive treatment & $19(5.3)$ \\
\hline
\end{tabular}

Values are presented as mean (range) or number (\%).

${ }^{a}$ Catheter directed thrombolysis with/without stent or surgical thrombectomy.

was one year for patients with unprovoked DVT and 6 months for those with provoked DVT. CDT was performed in 13 limbs, and iliac stenting was performed in 10 cases. Surgical thrombectomy was performed in 6 cases with phlegmasia cerulea dolens. No patient exhibited clinical symptoms or signs suggestive of massive PE or right heart failure. The location of DVT is summarized in Table 2.

The incidence of PE according to the DVT laterality is 
summarized in Table 3. PE was detected in 199 (55.9\%) of the 356 patients with DVT. PE developed in 78 (62.9\%) patients with right-sided DVT and in 121 (52.2\%) with left-sided DVT $(\mathrm{P}=0.052)$. The incidence of PE according to the proximal extent of the DVT is summarized in Table 4. The site of DVT most commonly associated with the

Table 2. The laterality and location of deep vein thrombosis (DVT)

\begin{tabular}{lcc}
\hline \multicolumn{1}{c}{ Location of DVT } & Left side $(\mathrm{n}=232)$ & Right side $(\mathrm{n}=124)$ \\
\hline Iliac vein & $131(56.5)$ & $25(20.2)$ \\
Femoral vein & $59(25.4)$ & $71(57.3)$ \\
Popliteal vein & $20(8.6)$ & $18(14.5)$ \\
Calf vein & $22(9.5)$ & $10(8.1)$
\end{tabular}

Values are presented as number (\%).

Table 3. Pulmonary embolism (PE) and PE score according to the laterality of DVT

\begin{tabular}{|c|c|c|c|}
\hline \multirow{2}{*}{$\begin{array}{l}\text { Artery involved } \\
\text { with PE (score) }\end{array}$} & \multicolumn{2}{|c|}{ Laterality of DVT } & \multirow{2}{*}{-P-value } \\
\hline & Left side $(n=232)$ & Right side $(n=124)$ & \\
\hline None (0) & $111(47.8)$ & $46(37.1)$ & - \\
\hline Subsegmental (1) & $35(15.1)$ & $14(11.3)$ & - \\
\hline Segmental (2) & $29(12.5)$ & $19(15.3)$ & - \\
\hline Lobar (3) & $21(9.1)$ & $15(12.1)$ & - \\
\hline Main (4) & $36(15.5)$ & $30(24.2)$ & 0.045 \\
\hline Cases with PE & $121(52.2)$ & $78(62.9)$ & 0.052 \\
\hline Median PE score & $1(0-2.0)$ & $2(0-3.0)$ & 0.012 \\
\hline
\end{tabular}

Values are presented as number (\%) or median (interquartile range). DVT, deep vein thrombosis; -, not available. development of PE was the left iliac vein (59/199, 29.6\%). The incidence of PE involving the main pulmonary artery was significantly lower in those with left-sided DVT (36

Table 4. Pulmonary embolism (PE) and PE score according to location of DVT

\begin{tabular}{lcccc}
\hline \multirow{2}{*}{ PE } & \multicolumn{4}{c}{ Location of DVT } \\
\cline { 2 - 5 } PE score & Iliac & Femoral & Popliteal & Calf \\
Left & $0(0-2)$ & $2(0-4)$ & $1(0-3)$ & $1(0-2)$ \\
Right & $1(0-3)$ & $2(0-4)$ & $1(0-2.25)$ & $0.5(0-2.25)$ \\
P-value & 0.320 & 0.342 & 0.654 & 0.889 \\
PE & & & & \\
Subsegmental & & & & \\
$\quad$ Left & $20(15.3)$ & $7(11.9)$ & $3(15.0)$ & $5(22.7)$ \\
$\quad$ Right & $2(8.0)$ & $9(12.7)$ & $2(11.1)$ & $1(10.0)$ \\
Segmental & & & & \\
Left & $15(11.5)$ & $8(13.6)$ & $2(10.0)$ & $4(18.2)$ \\
$\quad$ Right & $4(16.0)$ & $9(12.7)$ & $4(22.2)$ & $2(20.0)$ \\
Lobar & & & & \\
Left & $10(7.6)$ & $6(10.2)$ & $3(15.0)$ & $2(9.1)$ \\
Right & $3(12.0)$ & $9(12.7)$ & $2(11.1)$ & $1(10.0)$ \\
Main & & & & \\
Left & $14(10.7)$ & $16(27.1)$ & $4(20.0)$ & $2(9.1)$ \\
Right & $4(16.0)$ & $23(32.4)$ & $2(11.1)$ & $1(10.0)$ \\
P-value & 0.279 & 0.747 & 0.586 & 0.576 \\
\hline
\end{tabular}

Values are presented as median (interquartile range) or number (\%). DVT, deep vein thrombosis.

${ }^{a}$ Mann-Whitney U-test between left and right DVT. 'Linear-bylinear association.

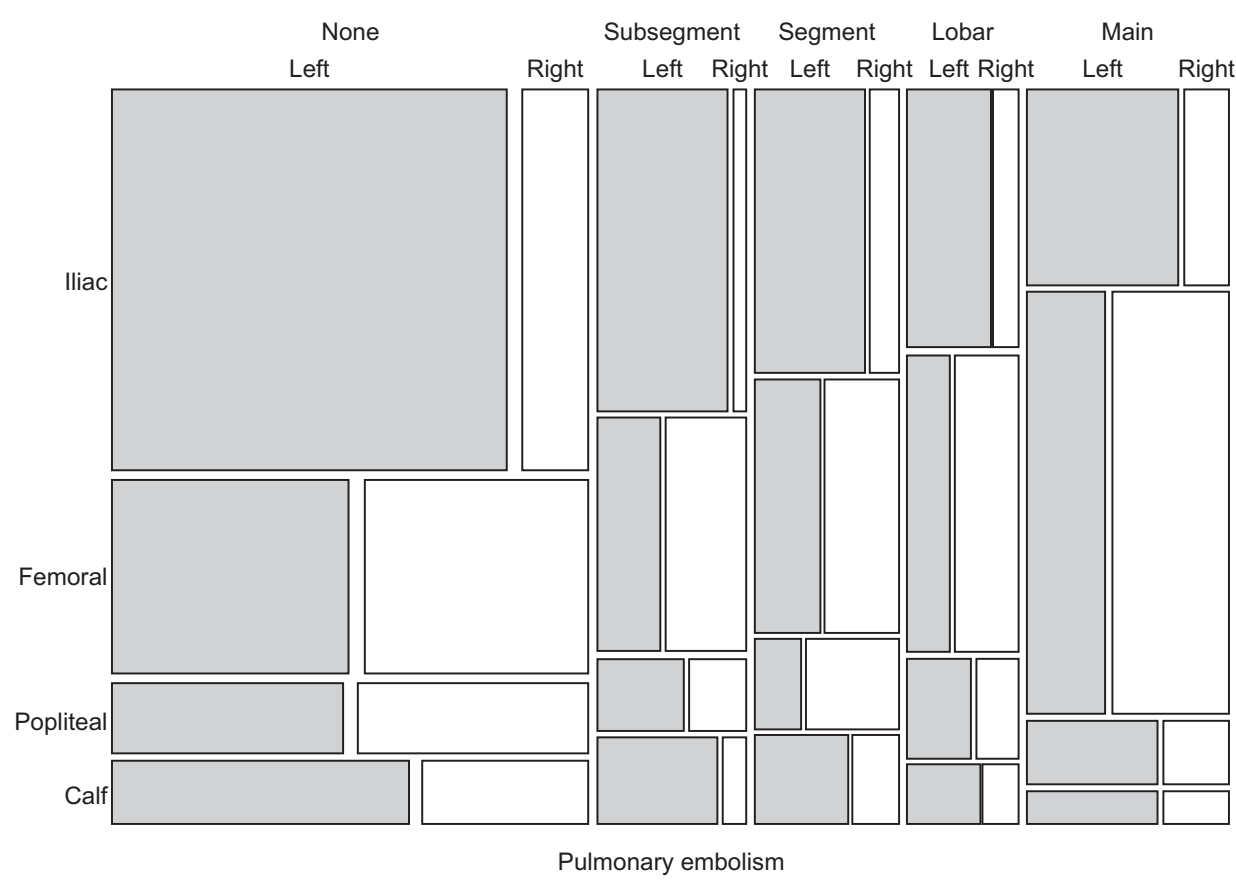

Fig. 2. Mosaic plot of pulmonary embolism, laterality, and proximal extent of deep vein thrombosis (DVT). Embolism of the main pulmonary artery developed more frequently in patients with DVT of the right femoral vein. 
cases, 15.5\%) than in those with right-sided DVT (30 cases, $24.2 \% ; P=0.045)$. When analyzed by the anatomical segment of the affected leg, PE occurred most frequently after DVT in the right femoral vein $(50 / 71 ; P=0.016)$. In addition, DVT of the right femoral vein had the highest rate of main pulmonary artery embolism ( 23 cases, $32 \% ; \mathrm{P}<0.001$ ). The median PE score was the highest in the right femoral veins (2.0, range $0-4)$.

lliac vein compression was observed in 63/131 cases (48.1\%) with left iliac vein thrombosis. Among those with DVTs in the left iliac vein, PE occurred more frequently among patients without iliac vein compression (36 cases, $52.9 \%$ ) than in those with iliac vein compression (23 cases, 36.5\%; $\mathrm{P}=0.043)$. Furthermore, $\mathrm{PE}$ involving the main pulmonary artery also occurred more frequently in patients without iliac vein compression (12 cases, 17.6\%) than in those with iliac vein compression ( 2 cases, $3.2 \%$; $\mathrm{P}=0.007$ ).

\section{DISCUSSION}

The primary concern in the treatment of acute DVT is the prevention of PE, in particular, fatal PE. DVTs of the lower extremity are a prevalent and major cause of PE; however, the laterality or location of DVT associated with the development of PE requires elucidation. This study showed a predominance of left-sided DVT, which was similar to the results of a previous study [17]. The incidence of left-sided DVT was about twice that of right-sided DVT; similarly, the incidence of PE was also more frequent on the left side over the right, and about 50\% of the leftsided DVTs were caused by an anatomical variant of iliac vein compression (63/131). In this observational study, leftsided DVTs were associated with a lower rate of PE than the right-sided DVTs. Additionally, in cases of PE involving the main pulmonary artery, the incidence of left-sided DVT was significantly lower than that of right-sided DVT. Analysis of the DVT location showed that the left iliac vein was the most frequent site of DVT; however, the rate of development of PE was higher in cases with DVT of the right femoral vein. In cases of PE in the main pulmonary artery, the right femoral vein was the most frequent site of origin (Fig. 2). These findings suggest that right-sided DVT has a higher frequency of causing PEs than the left-sided DVT, despite its relatively low incidence. Moreover, patients with left iliac vein compression syndrome had a lower overall rate of PE and a lower rate of PE in the main pulmonary artery than in those without iliac vein compression. Therefore, it can be assumed that the reason for the higher incidence of PE in patients with right-sided DVT is the underlying pathophysiology of the left-sided DVT. These results are similar to the results of previous studies, which showed that the rate of symptomatic PE in patients with left-side DVT is lower than that in those with right-sided DVT [15]. This can be partially explained by the protective effect of iliac vein compression against massive PE [18].

While some researchers have reported the left-sided predominance or anatomical distribution of DVT, reports about the incidence of PE related to the laterality and anatomical location of DVT are scarce. Some studies showed that iliac vein compression has a protective effect against PE $[18,19]$. However, to the best of our knowledge, this study is the first quantitative analysis of the incidence of PE according to the laterality and anatomical location of DVT. Our results indicate that patients with right-sided DVTs require more careful observation for the possible occurrence of main pulmonary artery PE. In our study, the incidence of PE (18.6\%) in cases with calf vein thrombosis was relatively higher than that in other studies (0\%-6.2\%) [20]. We believe that this is due to the difference in the method of diagnosis of PE, CT pulmonary arteriography or ventilation/perfusion scan.

There were some limitations in this study. First, the nonrandomized observational nature of this study has inherent selection bias because we only included patients who underwent a CT scan. Second, we did not analyze the previously known risk factors of PE; hence, the risk factors might be skewed. Finally, the underlying causes of the DVT, such as hypercoagulability or malignancy related to the PE, were not included in our analysis. Future studies investigating the incidence of PE based on the underlying causes of DVT are necessary to direct the further treatment modalities.

\section{CONCLUSION}

The incidence of left-sided leg DVT was about two times higher than that of right-sided DVT. However, PE of the main pulmonary trunks is more frequent in patients with right-sided DVTs. Hence, careful observation is necessary for patients with right-sided DVTs considering the possibility of main pulmonary artery PE.

\section{CONFLICTS OF INTEREST}

The authors have nothing to disclose.

\section{ORCID}

\author{
Sangmin Gong \\ https://orcid.org/0000-0002-8013-5903 \\ Eun Ji lee \\ https://orcid.org/0000-0001-8756-4287 \\ Jin Sung Kim \\ https://orcid.org/0000-0002-5927-4909
}




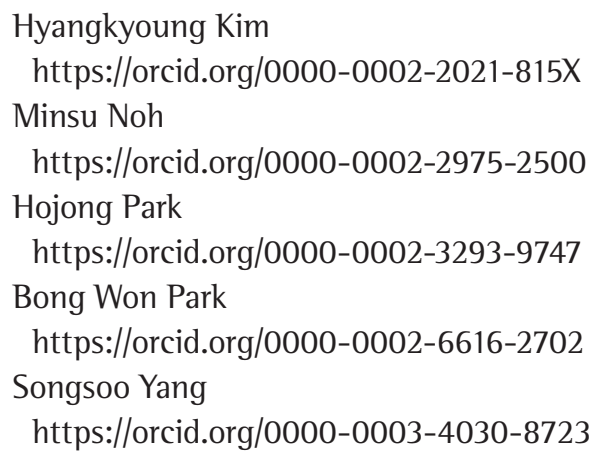

\author{
Sang Jun Park \\ https://orcid.org/0000-0001-8112-6331
}

\section{AUTHOR CONTRIBUTIONS}

Concept and design: SG, SJP. Analysis and interpretation: JSK, MN. Data Collection: SG, EJL. Writing the article: SJP. Critical revision of the article: HP, BWP, SY. Final approval of the article: All. Statistical analysis: HK. Obtained funding: none. Overall responsibility: SJP.

\section{REFERENCES}

1) Levine MN, Hirsh J, Gent M, Turpie AG, Weitz J, Ginsberg J, et al. Optimal duration of oral anticoagulant therapy: a randomized trial comparing four weeks with three months of warfarin in patients with proximal deep vein thrombosis. Thromb Haemost 1995;74:606-611.

2) Armon MP, Hopkinson BR. Thrombolysis for acute deep vein thrombosis. $\mathrm{Br}$ J Surg 1996;83:580-581.

3) Heymans S, Verhaeghe R, Stockx L, Collen D. Feasibility study of catheter-directed thrombolysis with recombinant staphylokinase in deep venous thrombosis. Thromb Haemost 1998;79:517-519.

4) Chen F, Den J, Yuan QW, Zhou WM, Xiong JX, Zhou W. Compression of left common iliac vein is independently associated with left-sided deep vein thrombosis. J Vasc Surg Venous Lymphat Disord 2013;1:364-369.

5) Kibbe MR, Ujiki M, Goodwin AL, Eskandari M, Yao J, Matsumura J. Iliac vein compression in an asymptomatic patient population. J Vasc Surg 2004;39:937-943.

6) O'Sullivan GJ, Semba CP, Bittner CA, Kee ST, Razavi MK, Sze DY, et al. Endovascular management of iliac vein compression (May-Thurner) syndrome. J Vasc Interv Radiol 2000;11:823-836.

7) Knipp BS, Ferguson E, Williams DM, Dasika NJ, Cwikiel W, Henke PK, et al.
Factors associated with outcome after interventional treatment of symptomatic iliac vein compression syndrome. J Vasc Surg 2007;46:743-749.

8) Narayan A, Eng J, Carmi L, McGrane S, Ahmed M, Sharrett AR, et al. lliac vein compression as risk factor for leftversus right-sided deep venous thrombosis: case-control study. Radiology 2012;265:949-957.

9) Shin HS, Park JK. The laterality of deep vein thrombosis in the pelvic and lower extremity veins. Vasc Specialist Int 2014;30:56-61.

10) Casella IB, Bosch MA, Sabbag CR. Incidence and risk factors for bilateral deep venous thrombosis of the lower limbs. Angiology 2009;60:99-103.

11) Mathur M, Cohen M, Bashir R. MayThurner syndrome. Circulation 2014;129:824-825.

12) Du GC, Zhang MC, Zhao JC. Catheterdirected thrombolysis plus anticoagulation versus anticoagulation alone in the treatment of proximal deep vein thrombosis- a meta-analysis. Vasa 2015;44:195-202.

13) Becattini C, Agnelli G, Lankeit M, Masotti L, Pruszczyk P, Casazza F, et al. Acute pulmonary embolism: mortality prediction by the 2014 European Society of Cardiology risk stratification model. Eur Respir J 2016;48:780-786.

14) Davies MG, El-Sayed HF. Current status of clot removal for acute pulmonary embolism. Ann Vasc Surg 2016;31:211220.

15) Wells PS, Anderson DR, Rodger M, Ginsberg JS, Kearon C, Gent M, et al. Derivation of a simple clinical model to categorize patients probability of pulmonary embolism: increasing the models utility with the SimpliRED Ddimer. Thromb Haemost 2000;83:416420.

16) Le Gal G, Righini M, Roy PM, Sanchez $\mathrm{O}$, Aujesky D, Bounameaux $\mathrm{H}$, et al. Prediction of pulmonary embolism in the emergency department: the revised Geneva score. Ann Intern Med 2006;144:165-171.

17) Ouriel K, Green RM, Greenberg RK, Clair DG. The anatomy of deep venous thrombosis of the lower extremity. J Vasc Surg 2000;31:895-900.

18) Jun H. Endovenous intervention of May-Thurner syndrome with thrombus beyond iliac vein stenosis. Vasc Specialist Int 2019;35:90-94.

19) Chan KT, Tye GA, Popat RA, Kuo WT, Unver K, Kothary N, et al. Common iliac vein stenosis: a risk factor for oral contraceptive-induced deep vein thrombosis. Am J Obstet Gynecol 2011;205:537.e1-e6.

20) Wu AR, Garry J, Labropoulos N. Incidence of pulmonary embolism in patients with isolated calf deep vein thrombosis. J Vasc Surg Venous Lymphat Disord 2017;5:274-279. 